\title{
Choice of Photodetectors for the Design of SACOCDMA System Employing Multi-Diagonal Code
}

\author{
Bhanu Priya \\ Department of Electronics and Communication Engineering \\ GNDU Regional campus, Jalandhar \\ Bpriya812@gmail.com
}

\begin{abstract}
Optical communication are difficult to design and analyze therefore it is useful to find the effects of various parameters and characteristics of the Photodetectors used in optical systems. So in this paper effect of various parameters on the performance of proposed system with both types of Photodetectors is analyzed. Through the simulative results taken from "Optisystem 11.0" it is concluded that APD has an edge over PIN at Lower data rates, at lower transmit power levels, at higher attenuation and can support large transmission distance but PIN is preferred at higher thermal noise and higher data rates in comparison to APD.
\end{abstract}

Keywords: Avalanche Photodiode (APD), PIN, Multi-Diagonal Code (MD)

\section{Introduction}

OCDMA systems have always been employed for local area networks and later for access network application [1-3] because of their inherent capability of dynamic bandwidth assignment, asynchronous access networks, and many more applications. But it is known that in OCDMA systems there exist PIIN and MAI. Codes with zero-cross correlation are proposed in order to remove the above mentioned drawbacks. One of them is Multi-Diagonal codes which is easy to design, support many users at high data rate and offers flexibility to choose weight parameters [4].

PIN is a semiconductor Photo-detector without internal gain which generates single electron-hole pair for absorbed photon and it can operate at longer wavelengths [5]. Avalanche photodiode internally multiplies the primary signal photocurrent before it enters the input circuitry. This phenomenon of carrier multiplication is called impact ionization [6]. The newly generated carriers are accelerated by the effect of high electric field thus absorbing enough energy to cause further impact ionization. This process is called avalanche effect.

\section{Simulation Setup}

Figure 1 shows block diagram of proposed system with two users with help of MD codes. In this design continuous wave Laser is used at transmitter side according to weight (i.e., 2 in this case). The data is modulated by the use of Mach Zehnder modulator. After it multiplexer is used to collect the signal from different users [7]. The tests were carried out at a rate of $10 \mathrm{~Gb} / \mathrm{s}$ for 10-km distance with the ITU-T G.652 standard singlemode optical fiber (SMF).All the attenuation $\alpha$ (i.e., $0.25 \mathrm{~dB} / \mathrm{km}$ ), dispersion (i.e., 18 $\mathrm{ps} / \mathrm{nm} \mathrm{km}$ ), and nonlinear effects were added At the receiver side Photo-Detector converts optical signal into electrical one whereas filtering action is provided by Low Pass Filter. Finally the results are visualized by BER analyzer [8]. 


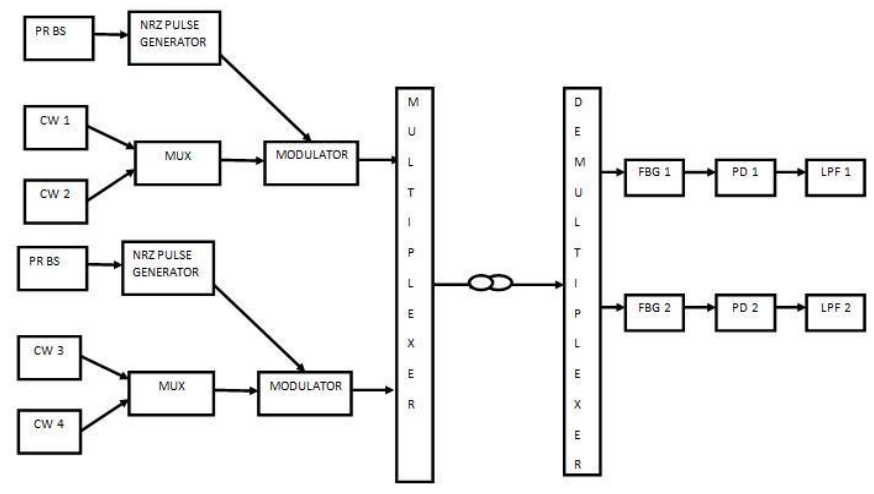

Figure 1. Block Diagram of SAC OCDMA-FSO System using MD Codes

\section{Results and Discussions}

Simulation software Optisystem 11.0 is used in order to evaluate the performance of the SAC-OCDMA system based on MD code with different Photodetectors i.e., APD and PIN. Simulation was carried out with different parameters to analyze the performance of proposed design. All the parameters value used during simulation is arranged in Table 1. presented below.

Table 1. Parameters used During Simulation

\begin{tabular}{|l|c|}
\hline \multicolumn{1}{|c|}{ Parameters } & Value \\
\hline $\begin{array}{l}\text { Operating } \\
\text { wavelength } \\
\text { region }\end{array}$ & $1550 \mathrm{~nm}$ \\
\hline Signal data & 128 -bit PN sequence \\
\hline Signal Bit Rate & $10 \mathrm{Gbps}$ \\
\hline Dispersion & $18 \mathrm{ps} / \mathrm{nm} \mathrm{km}$ \\
\hline Attenuation & $0.25 \mathrm{~dB} / \mathrm{km}$ \\
\hline
\end{tabular}

\subsection{Effect of Data Rate}

The following results were obtained at $0 \mathrm{dBm}$ (indicates that there exists no impairment due to channel between transmitter and receiver). At Lower data rates Avalanche Photodiode performs better than PIN but when the data rate is increased PIN performs better due to random nature of APD gain mechanism which leads to dominance of shot noise. As from Figure 2 it is clear that at data rate of 2.5 Gbps APD and PIN provides quality factor of 41.2558 and 27.1063 respectively. So from this difference it has been observed that data rate significantly affect BER Performance when using different type of receiver whether it is PIN or APD. Figure 3 depicting the eye diagram obtained with the use of two Photodetectors clears the priority towards APD as distortion are observed with the application of PIN. 


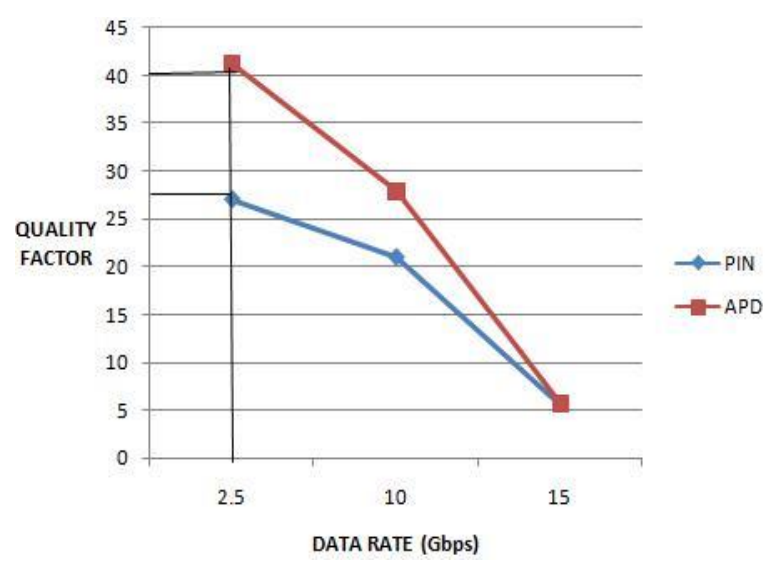

Figure 2. BER of Proposed System at Different Data Rates

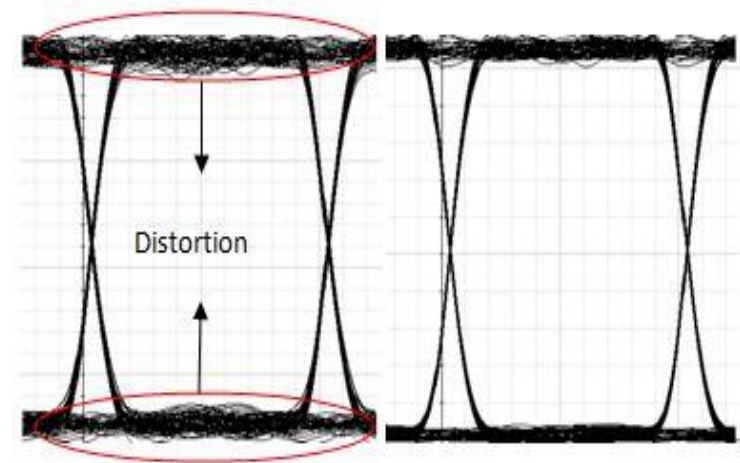

Figure 3. Eye Diagram of (a) PIN (b) APD at 2.5 Gbps

\subsection{Effect of Transmit Power Levels}

The Proposed system was simulated at 10Gbps to obtain the below mentioned results. As it is clearly predicted from the Figure 4 at lower transmit power levels APD is more sensitive as BER of the order of $10 \exp (-15)$ with quality factor of 7.87552 whereas PIN doesn't achieves BER within allowable limits at $-10 \mathrm{dbm}$ as transmit power level due to the internal gain generated in APD that increases the photocurrent by the multiplication factor. Figure 5 depicts that $\mathrm{h} 2>\mathrm{h} 1$ where $\mathrm{h} 1$ and $\mathrm{h} 2$ are the eye heights obtained with PIN and APD.With the application of PIN Jitter is observed because with lower transmit power levels the signal is unable to overcome the distortion. 


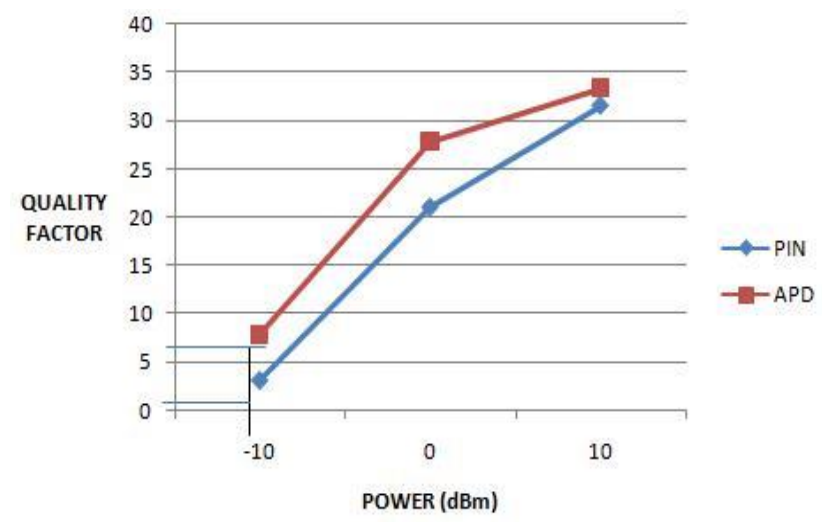

Figure 4. BER of Proposed System at Different Transmit Power Levels
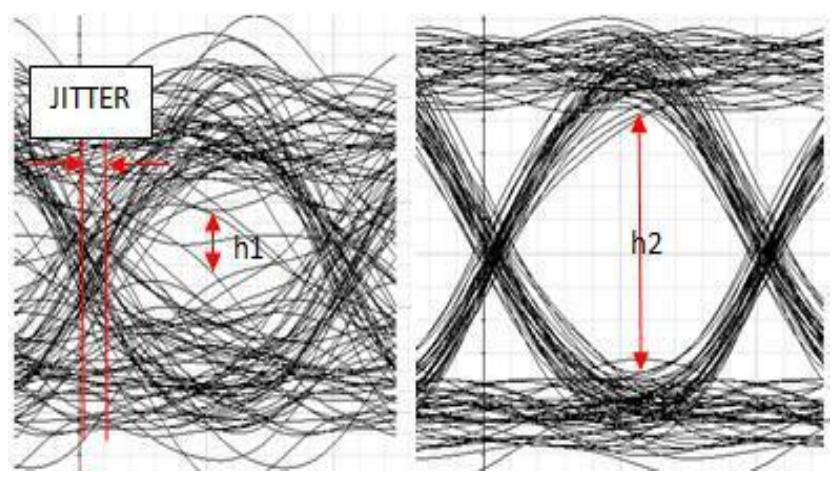

Figure 5. Eye Diagram of (a) PIN (b) APD at $-10 \mathrm{dBm}$

\subsection{Effect of Transmission Distance}

This design of SACOCDMA system employing multi-diagonal code was carried out 10 Gbps. With these conditions APD is able to support transmission distance upto $51 \mathrm{~km}$ in comparison to PIN which is successful upto $35 \mathrm{~km}$ as shown in Figure 6 The Eye diagram shown in Figure 7 depicts the efficiency of APD as a Photodetector as they show an internal current gain effect (around 100) due to impact ionization (avalanche effect) [9]. As concluded from the eye diagram $\mathrm{x} 2>\mathrm{x} 1$ whereas $\mathrm{x} 1$ and $\mathrm{x} 2$ are the eye heights obtained with PIN and APD so with help of APD the eye is open wide as compared to eye diagram of PIN which is distorted a bit. 


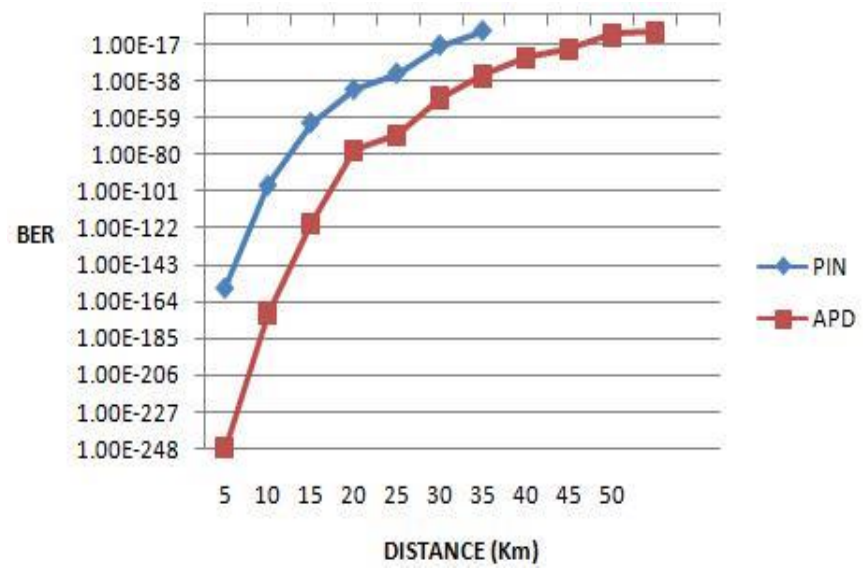

Figure 6. BER of Proposed System at Different Transmission Distance

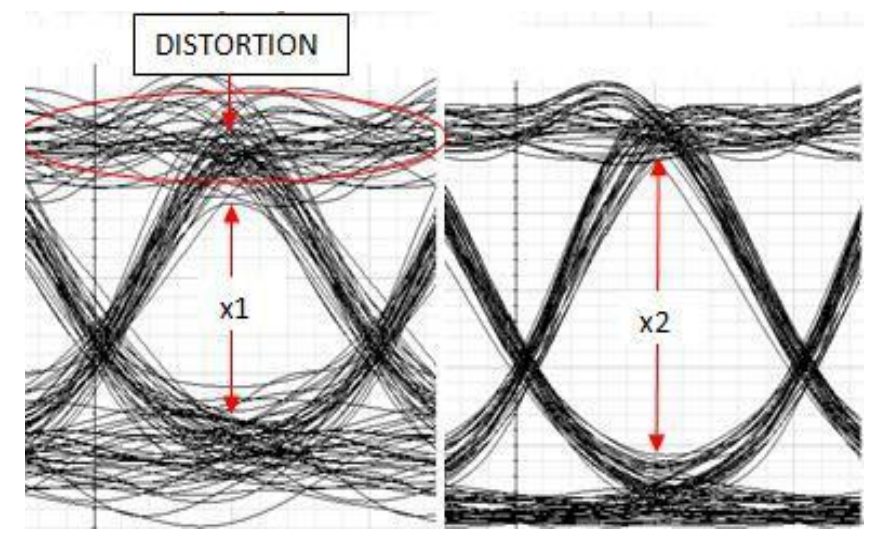

Figure 7. Eye Diagram of (a) PIN (b) APD at $35 \mathrm{~km}$

\subsection{Effect of APD Gain}

Gain optimization of APD has been demonstrated in Figure 8. The APD gain values which falls between the ranges of 3 to 4 is considered as perfect window to select APD gain values for the simulation of proposed system using MD codes [10].
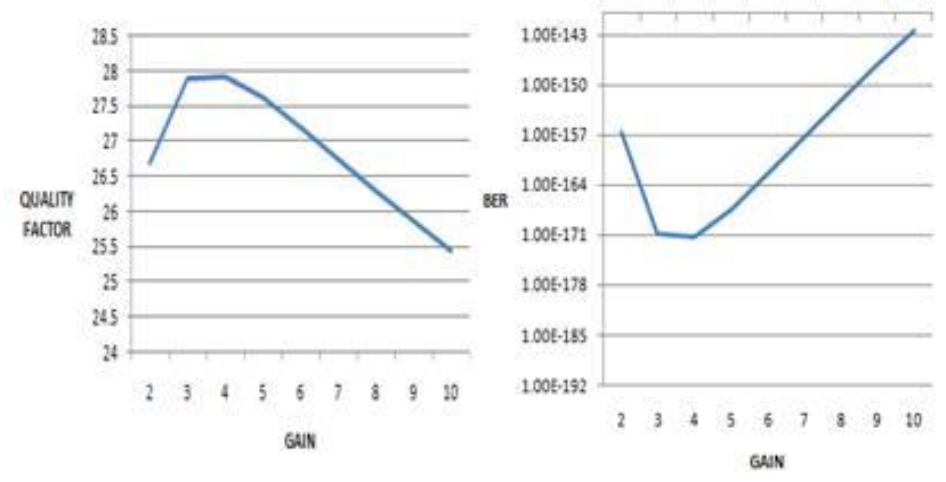

Figure 8. (a) Quality Factor (b) BER of Proposed System with APD at Different Gain 


\subsection{Effect of Thermal Noise}

At lower values of thermal noise obviously APD fails to be superior Over PIN, i.e., where shot noise is high. Whereas, in the APD, as shot dominates over thermal noise its performance becomes worse (Figure 9) due to high temperature existence and applied voltages in APD it is less stable than the PIN [11].

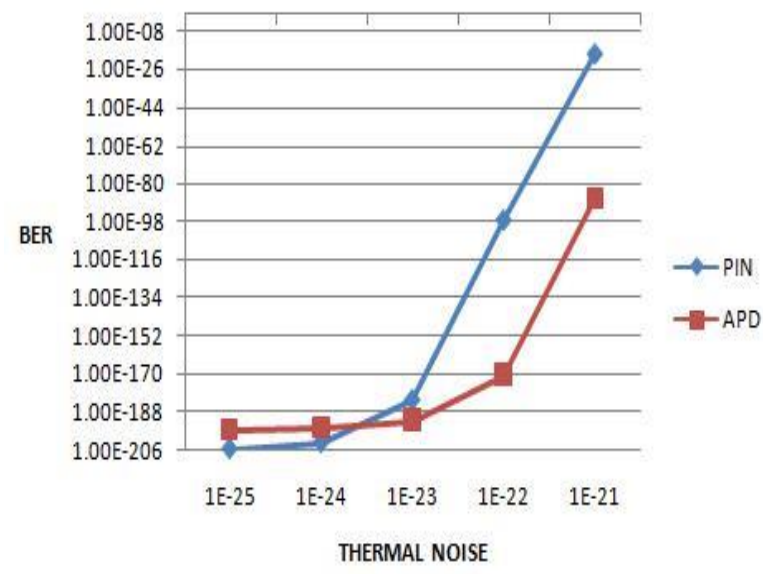

Figure 9. BER of Proposed System at Different Values of Thermal Noise

\subsection{Effect of Attenuation}

With increase in attenuation as shown in Figure 10 BER with both the Photodetectors increases but APD is capable to withstand attenuation upto $1.45 \mathrm{~dB} / \mathrm{km}$ and PIN upto $0.95 \mathrm{~dB} / \mathrm{km}$ due to the fact that APD internally multiplies the primary photocurrent by multiplication factor. The difference between the applications of two Photodetector is clearly depicted in Figure 11 as w2>w1 (more the width more the time to sample the data without error) so APD can detect the signal well even with increased attenuation.

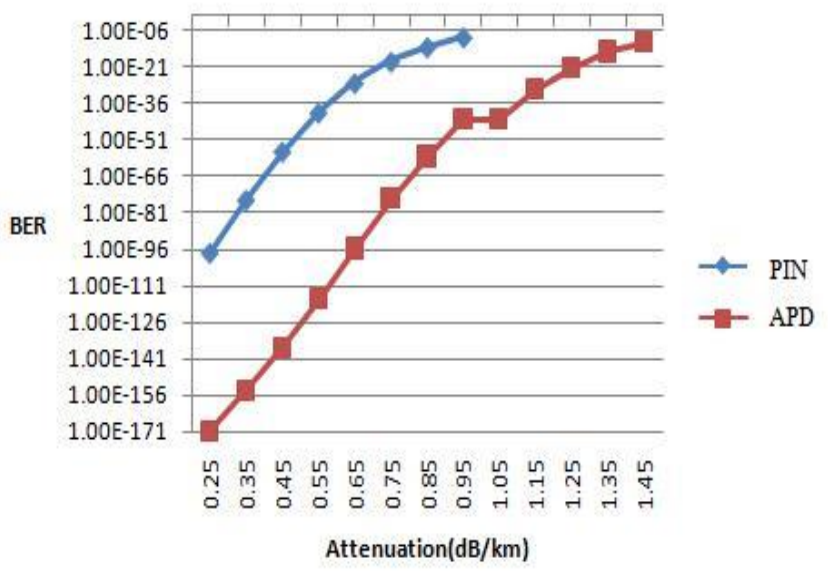

Figure 10. BER of Proposed System at Different Values of Attenuation 


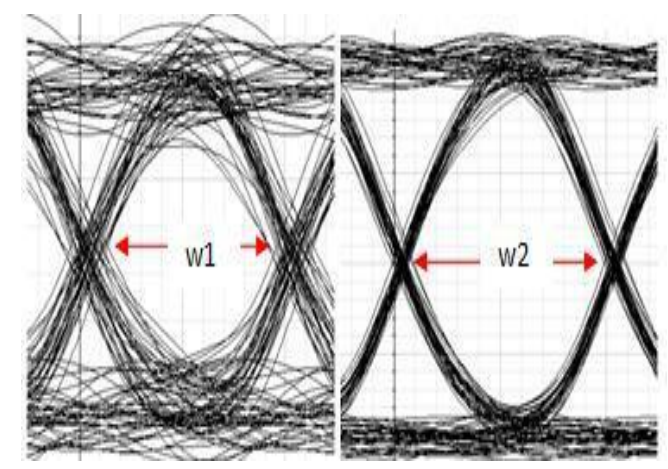

\section{Figure 11. Eye Diagram of (a) PIN (b) APD at $0.95 \mathrm{~dB} / \mathrm{km}$}

\section{Conclusion}

In this paper an attempt is made to extensively analyze the performance of SACOCDMA systems employing MD codes with PIN and APD. It is proposed how these Photo detectors can be utilized in proposed systems. Due to internal multiplication of photocurrent with multiplication factor APD is preferred over PIN at lower transmit power levels, higher attenuation, lower data rates, higher distances but due to higher temperature existence performance of APD get worsen at high thermal noise and larger data rates.

\section{References}

[1] M. R. Dale and R. M. Gagliardi, "Channel coding for asynchronous fiber optic CDMA communications", IEEE Trans. Commun, vol. 43, (1995), pp. 2485-2492.

[2] G. Lei Xu, I. Glesk, V. Baby and P. R. Prucnal, "Multiple access interference (MAI) noise reduction in a 2D optical CDMA system using ultrafast optical thresholding", 17th Annual Meeting of the IEEE, Lasers and Electro-Optics Society, vol. 2, (2004) November 8-9, pp. 591-592.

[3] W. Chen and S. Liu, "PIN avalanche photodiodes model for circuit simulation", IEEE Journal Quant. Electron, vol. 32, (1996) December, pp. 2105-2111.

[4] A. Ebberg, R. Bauknecht, M. Bittner, M. Grumm and M. J. T. Bitter, "High performance optical receiver module for $10 \mathrm{~Gb} / \mathrm{s}$ applications with low cost potential”, Electron. Lett., vol. 36, (2000) April, pp. 741-742.

[5] Z. Wei, "Modified quadratic congruence codes for fiber Bragg grating based spectral amplitude coding optical CDMA system", Journal Lightwave Technol, vol. 19, (2001), pp. 1274-1281.

[6] M. Rochette, S. Ayotte and L. A. Rusch, "Analysis of the spectral efficiency of frequency-encoded OCDMA system with incoherent sources”, Journal Lightwave Technol., vol. 23, (2005) April, pp. 16101619.

[7] H. Ghafouri-Shiraz Zou, "Unipolar codes with ideal in-phase cross-correlation for spectral amplitudecoding optical CDMA systems", IEEE Trans. Commun., vol. 50, (2002) August.

[8] M. R. Dale and R. M. Gagliardi, "Channel coding for asynchronous fiber optic CDMA communications", IEEE Trans. Commun., vol. 43, (1995), pp. 2485-2492.

[9] O. Kharraz and D. Forsyth, "PIN and APD photodetector efficiencies in the longer wavelength range 1300-1550 nm", Optik, vol. 124, (2013), pp. 2574-2576.

[10] M. S. Anuara, S. A. AlJunida, A. R. Arief a, M. N. Junitaa and N. M.Saadb, "PIN versus Avalanche photodiode gain optimization in zero cross correlation optical code division multiple access system", Optik, vol. 124, (2013), pp. 371-375.

[11] O. Kharraz and D. Forsyth, "Performance comparisons between PIN and APD photodetectors for use in optical communication systems", Optik, vol. 124, (2013), pp. 1493-1498. 
International Journal of Signal Processing, Image Processing and Pattern Recognition Vol.8, No.12 (2015) 